\title{
Feasibility of a Socially Intelligent Tutor
}

\author{
Jozef Tvarožek and Mária Bieliková \\ Faculty of Informatics and Information Technologies, \\ Slovak University of Technology, \\ Ilkovičova 3, 84216 Bratislava, Slovakia \\ \{jtvarozek, bielik\}@fiit.stuba.sk
}

\begin{abstract}
We present feasibility study of an intelligent tutoring system Peoplia in which a socially intelligent tutoring agent uses common instructional methods that are augmented by social features to help students learn. Peoplia features pseudo-tutor assessments, free-text answering, personalized question generation, and adaptive question selection. It allows students to work both individually and collaboratively while the tutoring friend monitors their social behavior and motivates them by socially relevant interventions.
\end{abstract}

Keywords: social intelligence, intelligent tutoring friend, motivation.

\section{Introduction}

Sustained student motivation is important for effective learning. However, providing motivational feedback is often at odds with cognitive scaffolding, and research is still seeking the right balance between the two [1]. In our research we attempt to improve students' motivation in a traditional tutoring environment using a socially intelligent agent, the tutoring friend, which addresses aspects beyond that of an individual student. A tutoring friend is an artificial learning companion that manages relationships with students, monitors their social behavior, and can provide them with interventions appropriate for the social context in which they learn [2]. All in all, research in politeness and its role in effective tutorial dialogue, motivating students and learning $[3,4]$ suggests that intelligent tutors can maintain the appearance of being socially intelligent by carefully selecting the appropriate words at the appropriate time, not requiring the presumably unavoidable labor intensive language processing methods. In our approach we attempt to follow these observations.

\section{The Peoplia System and Feasibility Study}

Peoplia is an interactive web-based environment that helps students to learn using various types of learning opportunities that are facilitated by a socially intelligent agent, the tutoring friend. It is a rework of our previous idea of a computerized assessment system that supports traditional classroom assessment [5] with 
an emphasis on problem solving, which is analogous to the Assistments system [6] adding a robust task generator that discourages cheating (during assessment) and surface approaches to learning (during exercise).

Problem solving. The central learning opportunity in Peoplia is problem solving. Students work on pseudo-tutor problems by attempting to solve a starting question (subtask) of the problem description, providing answers either textually or by interacting with an interactive component (e.g. radio button). In collaborative mode, individual problem solving is augmented with: (1) instant messaging, (2) voting for the most agreeable answer in the team, and (3) a multi-user interface. Student answers are graded (matched to the predefined set specified in the problem description) by the two-stage grading process with a human in the loop [5]. Course notes are enhanced to social study mode through the use of text highlights, sticky notes, and a dialogue facility for asking the tutoring agent or currently available fellow students for help.

Tutoring friend. The tutoring friend primarily manages the off-task social dialogue facility and does not participate directly in other learning activities which consequently appear for students to operate autonomously. The social support within the individual learning activities in Peoplia is strictly on structural level e.g. in the form of social recommendation of annotations, voting for best team answer, etc. as mentioned earlier in the descriptions of the learning activities. The tutoring friend influences the transitions between activities by a set of rules that can recommend a good course of action for the student at any given moment. The appearance of social intelligence in the tutoring friend is based on the data collected during the off-task social dialogues. The tutor's dialogue capability is scripted using an ignorant approach [7] enhanced by a dimensional model of relationship with the student [8].

Experiments. We conducted two experiments in middle school mathematics; in the first study, we were interested in how much would students revealed about themselves to an artificial friend that they never met before and that communicates via a text console, all under the assumption that they expect (after entering a computer lab for a math class) some form of computerized exercises or assessment. The tutoring friend was scripted to "go easy" on the student, politely ask how she feels, and inquire about her hobbies under the guise of providing her with personalized exercises.

16 students (6 females, 10 males) were transferred to the computer lab, and were instructed to work on math exercises in Peoplia. We were interested in the word count of students' comments in the welcome dialogue and the number of features (hobbies; such as to draw, sleep, watch TV, go out, and dog) they disclosed to the artificial tutoring friend. The mean word count per student was 11.625 (st.dev 8.69) and the mean feature count per student was 1.56 (st.dev 1.75), thus on average each student revealed at least one of her hobbies. However, $44 \%$ of the students ( 1 female, 6 males) ignored the welcome dialogue by not using more than 3 words; students that actually cooperated with the tutoring friend used 16.89 (st.dev 4.91) words, and revealed 2.78 (st.dev 1.39) features on average.

In the second study, 32 students (14 females, 18 males) took part in a 3 day long experiment in which first a pre-test was administered, then students took 2 instructional units (45 minutes) - one per day - followed by a post-test, with no 
instructional unit on the day of the post-test administration. The control group ( 8 females, 8 males) attended 2 units of traditional classroom instruction vs. 2 units of problem solving in Peoplia in the experimental group. We were interested how much students learned even though they "wasted" $10 \%$ of the available time for off-task interactions with an artificial agent, how this compares to traditional classroom instruction, and what differences would the interaction with social agent make. The unpaired $t$-test confirmed differences in the pre-test scores between the groups, and thus we cannot directly compare gains achieved by the experimental group vs. the control group. By analyzing only the results of the experimental group we get the $95 \%$ confidence interval for learning gains in the range $1.2 \%$ to $19.5 \%$, thus students in the experimental group did show nonzero learning, which was coincidentally at least as high (1.2\%) as in the control group (although not comparable).

We plan to repeat the study on a larger scale. In the questionnaire, students' feelings about how helpful the system was were modest, students tend to feel more positive about using the system again, and liking the system in general. When we filter out the 7 students who did not engage with the social agent we see fewer tasks attempted while solving more correctly, and also the questionnaire answers shift to the positive end. Students that did engage with the tutoring friend liked the system and the tutor more, and were also more successful in solving problems within the tutoring environment.

Acknowledgments. This work was supported by the Scientific Grant Agency of SR, grant No. VG1/0508/09, the Cultural and Educational Grant Agency of SR, grant No. 028-025STU-4/2010, and it is a partial result of the Research \& Development Operational Program for the project Support of Center of Excellence for Smart Technologies, Systems and Services II, ITMS 25240120029, co-funded by ERDF.

\section{References}

1. Boyer, K.E., Phillips, R., Wallis, M., Vouk, M., Lester, J.C.: Balancing the cognitive and motivational scaffolding in tutorial dialogue. In: ITS 2008, Montreal, Springer (2008)

2. Tvarožek, J., Bieliková, M.: The Friend: Socially-Intelligent Tutoring and Collaboration. In: AIED2009, IOS Press, Brighton, UK, pp.763-764 (2009)

3. Mayer, R.E., Johnson, W.L., Shaw, E., Sandhu, S.: Constructing computer-based tutors that are socially sensitive: Politeness in educational software. International Journal of HumanComputer Studies. 64(1), pp.36-42 (2006)

4. McLaren, B.,M., Lim, S., Yaron, D., Koedinger, K.R,: Can a Polite Intelligent Tutoring System Lead to Improved Learning Outside of the Lab? In: AIED2007, pp.331-338 (2007)

5. Tvarožek, J., Kravčík, M., Bieliková, M.: Towards Computerized Adaptive Assessment Based on Structured Tasks. In: Adaptive Hypermedia 2008, LNCS 5149, Springer, pp. 224 234 (2008)

6. Feng, M., Heffernan, N.T., Koedinger, K.R.: addressing the Testing Challenge with a WebBased E-Assessment System that Tutors as it Assesses. In: WWW 2006, New York, ACM Press, pp. 307-316 (2006)

7. McCalla, G. I., Murtagh, K.: G.E.N.I.U.S.: An experiment in ignorance-based automated program advising. AISB Newsletter, 75, UK, pp.13-20 (1991)

8. Svennevig, J.: Getting Acquainted in Conversation, John Benjamins, Philadelphia (1999) 